\title{
Unintended pregnancy with subdermal implant following miscarriage: response from the CEU
}

Thank you for the opportunity to comment on Dr Rank's letter. ${ }^{1}$ From her description of the case I calculate that the patient must have conceived 2 weeks after the first scan indicating possible miscarriage and attended for implant insertion before a urine pregnancy test would have been positive.

This case illustrates two pitfalls that can lead to missed diagnosis of pregnancy when starting contraception, namely wrongly assuming that vaginal bleeding is a menstrual period, and not taking account of pregnancy risk from unprotected sexual intercourse (UPSI) occurring within the pregnancy test 'window period'. These pitfalls can be difficult to avoid if vaginal bleeding is similar to the woman's usual period and occurs at the expected time or if the woman is questioned about UPSI but chooses not to disclose it. Faculty guidance acknowledges this uncertainty in advising that it is sufficient for a clinician to be reasonably sure that a woman is not pregnant before starting contraception. ${ }^{2}$

Dr Rank proposes two strategies to reduce the risk of missing a pregnancy. Starting contraception on the second day of bleeding might reduce the risk but it would not eliminate it completely, and I am not aware of any evidence to support routine Day 2 start over Day 1.

I would agree with Dr Rank's alternative suggestion of advising a pregnancy test 3 weeks after starting contraception if the bleed is shorter than usual. Faculty guidance does not currently include this as a specific recommendation but it would be in line with the Clinical Effectiveness Unit's Quick Starting Contraception guidance $^{2}$ and the criteria for being reasonably sure that a woman is not pregnant. ${ }^{2}$ We will consider including this suggestion as a good practice point in future guidance.

Louise Melvin, MRCOG, MFSRH

Director, Clinical Effectiveness Unit, Sandyford, Glasgow, UK; louise.melvin@nhs.net

Competing interests None 
J Fam Plann Reprod Health Care 2012;38:65-66.

doi:10.1136/fprhc-2011-100239

\section{REFERENCES}

Rank K. Unintended pregnancy with subdermal implant following miscarriage [Letter]. J Fam

Plann Reprod Health Care 2012:38:65.

2 Faculty of Sexual \& Reproductive

Healthcare Clinical Effectiveness

Unit. Quick Starting Contraception. 2010

http://www.fsrh.org/pdfs/CEUGuidance

QuickStartingContraception.pdf [accessed

14 October 2011] 